\title{
The UV upturn phenomenon in the hierarchical universe
}

\author{
Jaehyun Lee ${ }^{1,2}$ and Sukyoung K. Yi ${ }^{1}$ \\ ${ }^{1}$ Department of Astronomy \& Yonsei University Observatory, Yonsei University, \\ Seoul 120-749, Republic of Korea \\ email: syncphy@galaxy.yonsei.ac.kr
}

\begin{abstract}
Recent studies show that an old stellar population with high metallicity in the monolithic paradigm can explain the UV upturn. Numerical simulations and empirical studies however point out that massive early-type galaxies have evolved hierarchically with an extended star formation history. This obviously has an impact on our traditional understanding on the UV upturn and requires a new investigation on its origin. We report on our investigation on the evolutionary history of model galaxy SEDs in the hierarchical scenario. The use of conventional population models (calibrated to the monolithic picture) in combination with merger trees and extended star formation fails to reproduce the observed UV upturn. If a hierarchical picture is thought to be more realistic than a monolithic one, new calibration on the population models is required.
\end{abstract}

Keywords. galaxies: evolution, ultraviolet: galaxies

\section{Introduction}

The ultraviolet (UV) upturn phenomenon is one of the interesting features in elliptical galaxies. It has been believed that most of elliptical galaxies are composed of old stellar populations which are dim in the UV, and thus the phenomenon has attracted much attention. However, recent studies revealed that a considerable fraction of elliptical galaxies show residual star formation (RSF) (Yi et al. 2005), and that even old horizontal branch stars can produce UV light (e.g., Greggio \& Renzini 1990; Buzzoni 2007). Since the UV excess from young stellar populations shows a different SED morphology, it is likely that the UV upturn has the origin of extended horizontal-branch stars.

Numerical simulations and empirical studies show that elliptical galaxies are formed in a hierarchical buildup (e.g., Toomre \& Toomre 1972; Negroponte \& White 1983) involving mergers even recently (van Dokkum 2005). In this picture elliptical galaxies have a complex evolutionary history and various stellar sub-populations.

The UV upturn phenomenon provides a good constraint for stellar population and galaxy formation models. Yi, Demarque, \& Oemler (1998) shows that the SED of an old stellar population model with high metallicity in the monolithic paradigm shows a good agreement with the empirical data from the UV upturn of early-type galaxies. Going a step further, in this study, we look into the SEDs of model galaxies in the hierarchical universe.

\section{Model}

Since the semi-analytic approach was first introduced by White \& Frenk (1991), the semi-analytic model has become a good way to investigate galaxy formation and evolution. In this study, SEDs of galaxies are derived, based on our semi-analytic model. 

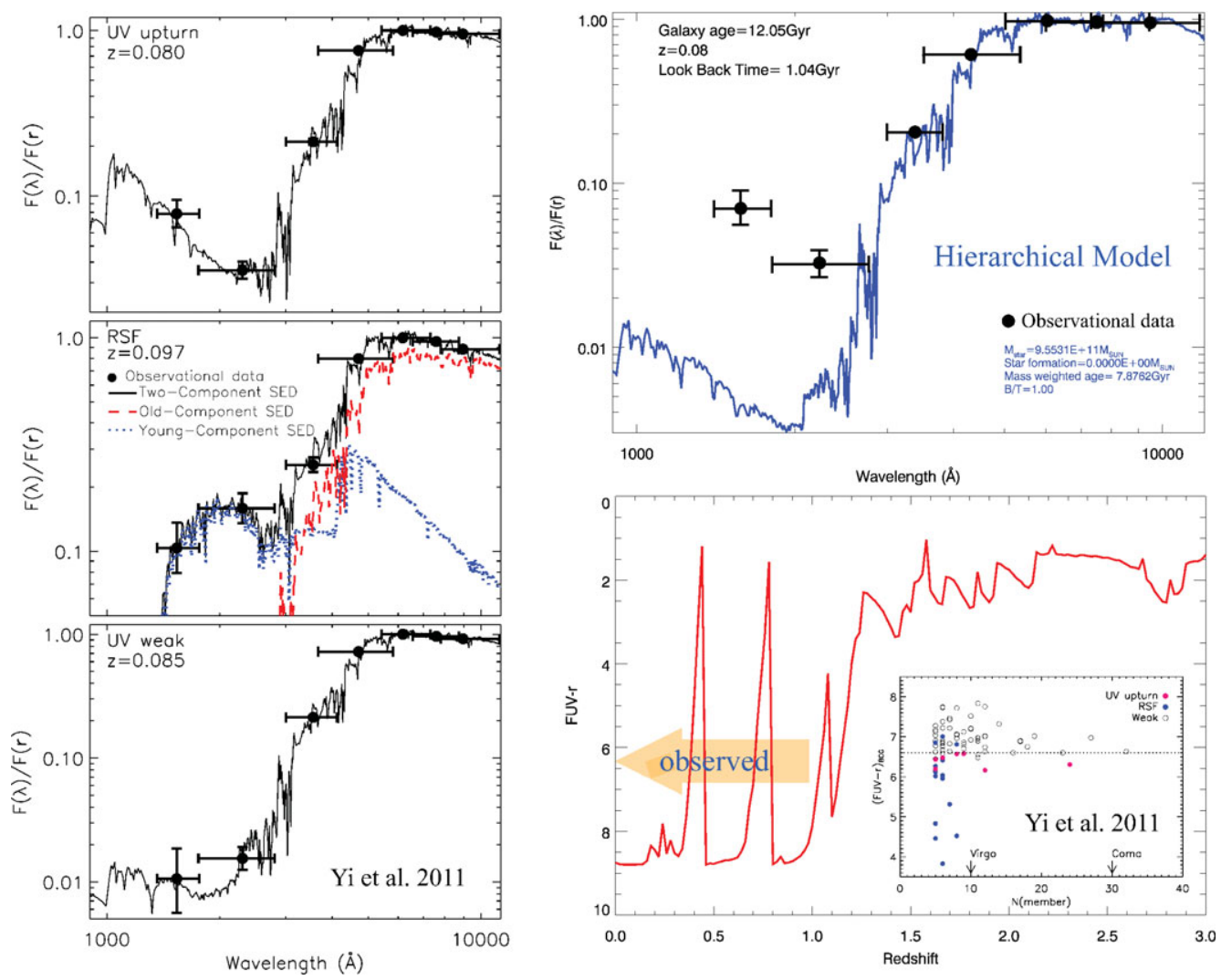

Figure 1. Left: Spectral fits for UV upturn, RSF, and UV weak galaxies. Upper right: SED of a model galaxy in the hierarchical scenario. Bottom right: FUV-r evolutionary history of the model galaxy.

We have developed a new semi-analytic model by considering accurate merging time scales, ram pressure and tidal stripping, merger-induced starburst, stellar mass loss and its recycling, and hot gas components of satellite galaxies.

The model SED library in this study is based on an aggregation of Yi (2003) and Bruzual \& Charlot (2003)(hereafter BC03). The Yi models are calibrated to trace the UV spectral evolution of old populations while the BC03 models cover a wide age range.

The evolutionary histories of galaxies in a $10^{14} \mathrm{M}_{\odot}$ model cluster are calculated by our semi-analytic model based on the extended Press-Schechter formalism. Since our semi-analytic model cannot consider chemical evolution yet and it is suggested that the UV upturn is mainly caused by metal-rich stellar populations, we assume super-solar metallicity $(Z=0.04)$ for all stellar populations in the model galaxies.

After calculating the evolutionary histories of model galaxies, the model SEDs of galaxies are synthesized by combining star formation histories and the model SED library.

\section{Results}

In Yi et al. (2011), a metal-composite stellar population with a simple old-age model can reproduce the UV upturn phenomenon while RSF and passive early-type galaxies show different SED morphology (Fig. 1). On the other hand, the SED of a typical massive 
early-type model galaxy from the hierarchical scenario cannot reproduce the UV upturn even though its metallicity is super solar $(\mathrm{Z}=0.04)$, as shown in the upper right panel.

The FUV-r of the model galaxy evolves as redshift decreases(bottom right panel). At high redshift, when the galaxy evolves with active star formation, the FUV-r is even less than 0. At low redshift, on the other hand, it rapidly increases notwithstanding that several starbursts change the color occasionally. The FUV-r of the model galaxy at $\mathrm{z}=0$ is roughly 9 , which is much redder than found in UV upturn or other passive elliptical galaxies.

A weakness of this study is that our model does not yet consider chemical evolution consistently while the UV upturn is sensitive to the metallicity.

\section{Discussion}

Until today, population studies on the UV upturn have been based on the monolithic formation scenario. In this simplistic picture, elliptical galaxies may develop a strong UV flux from hot HB stars. This has been a classical view. In the more realistic hierarchical scenario, however, giant elliptical galaxies grow with many mergers and hence likely have extended star formation histories and thus have smaller mass-weighted ages. For this reason, our attempt to reproduce the observed UV upturn using the traditional population models (calibrated for the monolithic "old" ellipticals) in combination with hierarchical halo merger trees bluntly failed. Thus, population models should be recalibrated to be effective and meaningful for UV upturn studies in the hierarchical merger picture.

\section{References}

Bruzual, G. \& Charlot, S. 2003, MNRAS, 344, 1000

Buzzoni, A. 2007, ASPC , 374, 311

Greggio, L. \& Renzini, A. 1990, ApJ , 364, 35

Negroponte, J. \& White, S. D. M. 1983, MNRAS, 205, 1009

Toomre, A. \& Toomre, J. 1972, BAAS , 4, 214

van Dokkum, P. G. 2005, AJ, 130, 2647

White, S. D. M. \& Frenk, C. S. 1991, ApJ, 379, 52

Yi, S. K., Demarque, P., \& Oemler, A. Jr. 1998, ApJ, 492, 480

Yi, S. K. 2003, ApJ, 572, 202

Yi, S. K. et al. 2005, AJ, 619, 111

Yi, S. K., Lee, J., Sheen, Y.-K., Jeong H., Suh, H., \& Oh, K. 2011, ApJS, 195, 22 